\title{
Photoacoustic effect applied to sound speed measurement
}

\section{Mayo Villagran-Muniz, Rosalba Castaneda-Guzman, Vicente Torres-Zuniga}

Mayo Villagran-Muniz, Rosalba Castaneda-Guzman, Vicente Torres-Zuniga, "Photoacoustic effect applied to sound speed measurement," Proc. SPIE 4588, Seventh International Conference on Education and Training in Optics and Photonics, (28 May 2002); doi: 10.1117/12.468720

SPIE Event: Education and Training in Optics and Photonics 2001, 2001, Singapore, Singapore 


\title{
Photoacoustic effect applied to sound speed measurement.
}

\author{
M. Villagrán-Muniz, R. Castañeda-Guzmán, V. Torres-Zúñiga \\ Laboratorio de Fotofísica, Centro de Instrumentos UNAM
}

Keywords: Photoacoustic effect, homemade laser, speed of sound.

\begin{abstract}
.
We present a simple experiment to show the photoacoustic effect, a well established but not widely known effect which has many applications. The photoacoustic effect consists of the generation of acoustic waves by pulsed radiation incident on a sample. In our case, we used a homemade Nitrogen laser as a source of pulsed light for many samples in order to measure the speed of sound. The Nitrogen laser is easy to build by undergraduate students, it is a transversal discharge laser at atmospheric pressure (TEA), excited by a Blumlein circuit, emitting nanosecond pulses in the ultraviolet region of the spectrum $(\lambda=337.1 \mathrm{~nm})$ and has been previously reported. The acoustic waves generated on the surface of the samples travel through the material and are detected with a piezoelectric sensor. The transducer is also easy to build using the piezoelectric of cigar lighters. The electric signals are registered by a $100 \mathrm{Mhz}$ oscilloscope triggered by the light produced at the laser discharge. Knowing the thickness of the sample and the arrival time of the acoustic wave we can precisely measure the speed of sound.
\end{abstract}

\section{INTRODUCTION.}

The speed of sound $\mathbf{c}$ is a characteristic property of a material, dependent on the thermodynamic variables of temperature $(\mathrm{T})$, pressure (p) and density $(\rho)$. The ordinary acoustic wave in a fluid corresponding to those normally audible to the human ear is independent of frequency. The theoretical value for the speed of sound in air at $0{ }^{\circ} \mathrm{C}$ is : $\mathbf{c}=331.6 \mathrm{~m} / \mathrm{s}$

The theoretical prediction of the speed of sound for liquids and solid materials is considerably more difficult than the gases. However, it is possible to show theoretically that for longitudinal waves on bars the speed of sound is expressed by ${ }^{1}$ :

$$
c=\sqrt{\frac{Y}{\rho}}
$$

where Y is Young's modulus or modulus of elasticity, characteristic of the material.

The photoacoustic effect was discover by A. Bell ${ }^{2}$ in 1880 and consists in the generation of sound waves in a material caused by the excitation with pulsed or modulated light.

In the case of using pulsed lasers, ultrasonic waves are generated, that can be detected with piezoelectric transducers. In our research laboratory, we have used this technique to characterize properties of diverse materials ${ }^{3-5}$.

On the other hand, in our teaching laboratory, low cost pulsed $\mathrm{N}_{2}$ lasers emitting in the ultraviolet region of the spectrum have been built, and an immediate application is to measure the speed of the sound in different materials such as metals, polymers, inorganic materials, and material compounds applying a photoacoustic method.

Currently, there are many the diverse techniques based on the photoacoustic effect and it is one of the more active research and technological development areas. 
The main idea of this work is to present a simple experiment, using the photoacoustic effect, and a homemade Nitrogen laser, to measure the speed of sound in solid materials. This experiment can be accomplished at the undergraduate level and with a low budget.

\section{NITROGEN LASER.}

The nitrogen laser is a very special type of gas laser. First, the lifetime of the upper laser level is shorter than the lower one. This property does not allow the $\mathrm{N}_{2}$ laser to operate in the continuous mode. Therefore, the $\mathrm{N}_{2}$ laser can only be operated in the pulsed mode where the maximum width of the pulse is limited by the upper laser level lifetime and it is about $5 \mathrm{~ns}$. Nitrogen molecules are excited by gas discharge produced from a short but fast rise time electrical pulse. The excited molecules will relax to the lower state by radiation producing light at a wavelength of 337 nanometers, which is not visible. However it could be detected through a white sheet paper that fluoresces with the light producing a violetblue spot on it. Besides this, the gain coefficient of the $\mathrm{N}_{2}$ laser is very high. Nitrogen lasers are also known as "superradiant" lasers. This type of laser is operated without any optical resonator. It has been used extensively as a pumping source for dye lasers ${ }^{6}$ and in photochemistry.

The experimental setup of the homemade nitrogen laser is shown in Figure 1. The system consists of a low inductance Blumlein transmission line, that can be easily made with tree flat plates of aluminum and an insulator (dielectric) forming two condensers $(\mathrm{C} 1, \mathrm{C} 2)$ with a common base plate, see Figure 1. Over the plates there are two cylindrical parallel electrodes separated by about $3 \mathrm{~mm}$ and connected by a coil (L). The condensers are charged through a high rectified voltage source, so that both reach the same potential. When the voltage reaches the air breakdown, a spark jumps between the electrodes exciting the gas, inducing the population inversion and producing laser radiation. The construction details and characterization of this type of laser can be found in the reference ${ }^{7}$.

Figure 1 shows the scheme of the electrical circuit of the $\mathrm{N}_{2}$ laser and in Table 1 the principal characteristics of this laser are presented.

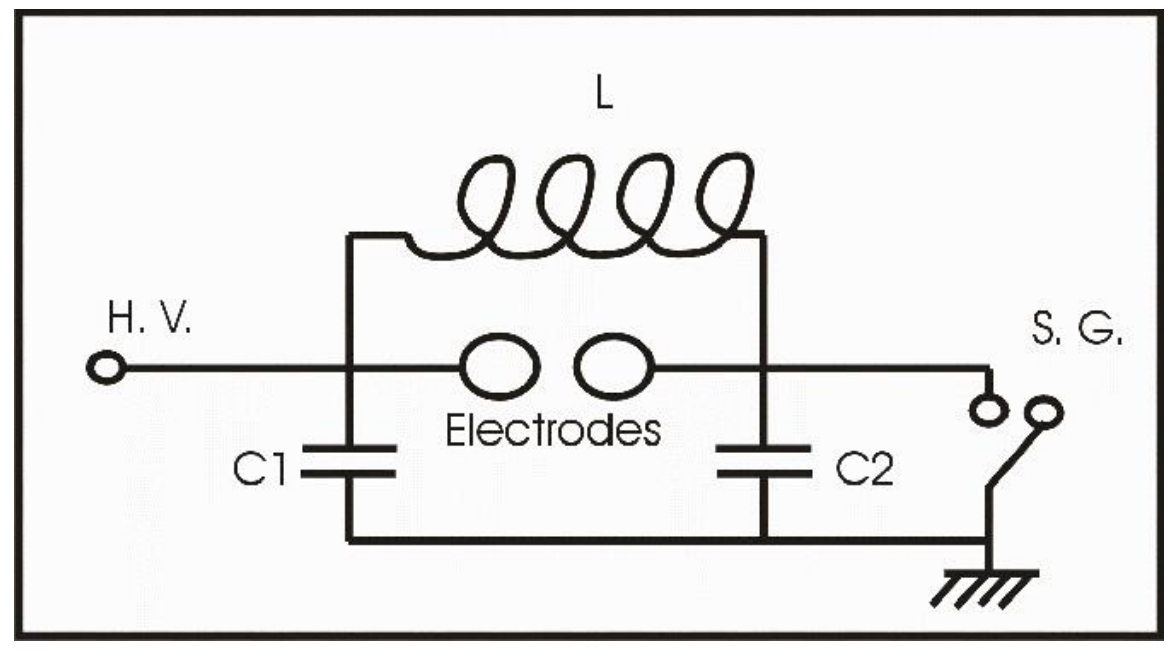

Figure 1. Scheme of Nitrogen laser. H. V. = high voltage, L = coil , S.G. = spark gap, C = capacitors

As we said, this laser was used to measure the speed of the sound in different materials with a photoacoustic technique, but any pulsed laser can be used in the following experiment; for example Nd:YAG laser, which is now a very common laser in many research and teaching laboratories. 
TABLE I. Characteristics of the $\mathrm{N}_{2}$ laser.

\begin{tabular}{|c|c|}
\hline CHARACTERISTICS & SPECIFICATIONS \\
\hline Peak Power & $76 \mathrm{~kW}$ \\
\hline Pulse Width & $2.9 \mathrm{~ns}$ \\
\hline Energy per Pulse & $220 \mu \mathrm{J}$ \\
\hline Repetition Rate & $1-20 \mathrm{~Hz}$ \\
\hline 9Average Power & $4.4 \mathrm{~mW}$ \\
\hline Wavelength & $337.1 \mathrm{~nm}$ \\
\hline Bandwidth & $0.5 \mathrm{~nm}$ \\
\hline Dimensions of the beam & $1 \times 3 \mathrm{~mm}$ \\
\hline Divergence of the beam & 4 mrad. wide \\
& 22 mrad. length \\
\hline
\end{tabular}

\section{PHOTOACOUSTIC METHOD.}

The photoacoustic technique using pulsed lasers has become a new experimental tool for material characterization ${ }^{3}$. This technique uses a mechanical wave generated on the surface of the material that travels at the speed of sound and is detected by using a piezoelectric transducer. The important advantages of this method are ${ }^{4-5}$ that special sample preparation is not required and no signal amplification is required due to the high signal to noise ratio obtained with piezoelectric transducers.

In photoacoustic experiments the interaction between the laser beam and the lattice produces an acoustic signal PA(t) $)^{5}$ which contains phenomenological information of the material. PA refers to the photoacoustic nature of the signal. And the index $\mathrm{t}$ indicates that PA is function of time. Once the signals are displayed on an oscilloscope, it is possible to measure the arrival time, or, they can be mathematically treated to extract the important physical information to obtain for example, phase transitions if the experiment is performed with a temperature variation ${ }^{8}$.

As the photoacoustic signal is generated on the surface of the sample, and travels at the characteristic speed of sound of the material, we only need to know the thickness of the sample and the time required to travel in it.

\section{EXPERIMENTAL SET UP.}

Figure 2 presents an experimental scheme for the photoacoustic method where the homemade Nitrogen laser is used to excite a sample coupled to a piezoelectric sensor. The transducer is also easy to build and a schematic diagram is presented in figure 3. We used a piezolectric ceramic as it is a sensor that can be obtained from a cigarette lighter. We put it in a metallic cartridge shell, with a BNC connector, that is connected directly to an oscilloscope. In the diagram we also show a lead block and another of copper, the first one is to suppress the rebounds of the acoustic wave and the second one is to couple a spring that is maintained tight and makes a good acoustic coupler to the sensor.

We used a quartz lens to focus the beam on the sample to increase the energy density. If the laser used has sufficient energy, it is not necessary to focus and also no quartz lens is required. It is necessary to ensure that the acoustic signal measured is synchronized with each pulse of the laser. Therefore, we used a photodetector that sensed part of the laser light through a beam splitter, and was connected to one of the channels of a $100 \mathrm{Mhz}$ oscilloscope as a trigger source. This reference signal will be our zero in time with respect to the arrive of the acoustics signal.

High voltage pulses produce electromagnetic noise. So a Faraday cage should be used in order to avoid problems in the signal displayed at the scope. In the case that a Faraday cage is not available, as the electromagnetic noise appears at the beginning of the signal, a wave-guide for the acoustic wave measured can be used. 


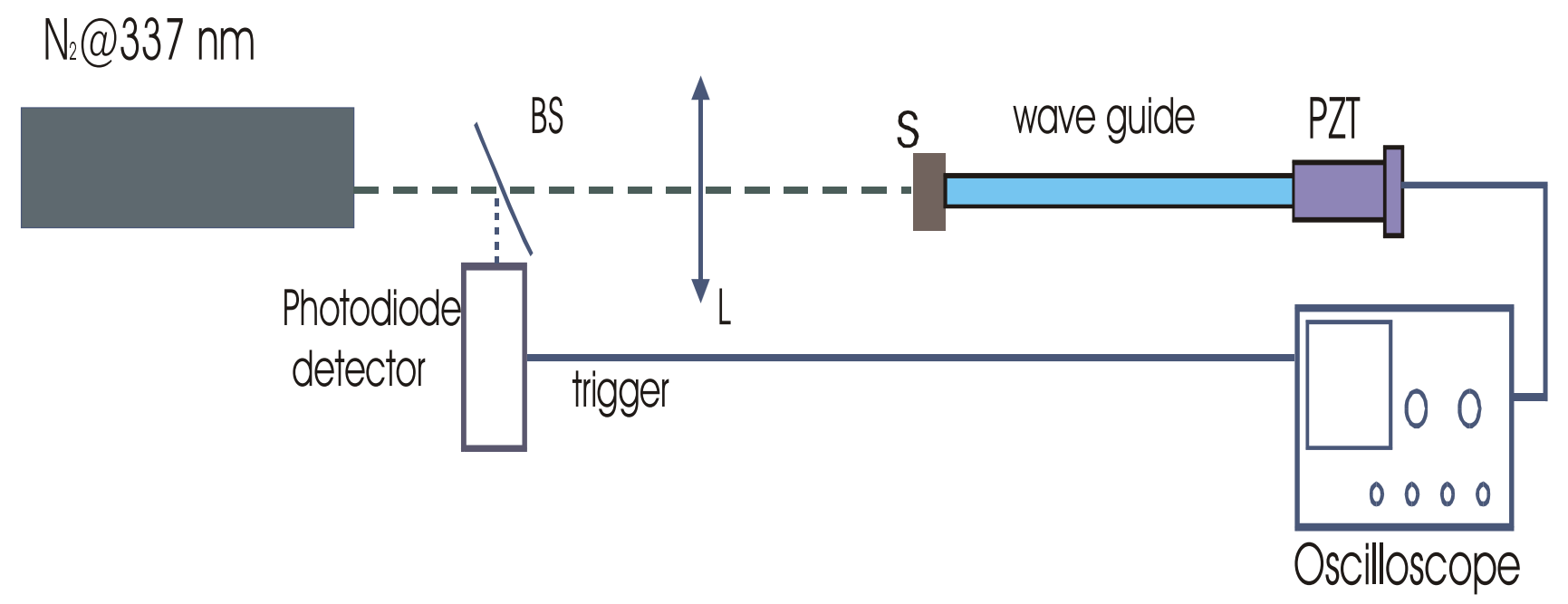

Figure 2. Experimental set up employed for the photoacoustic technique. $\mathrm{L}=$ Lens, $\mathrm{BS}=$ beam splitter, $\mathrm{PZT}=$ piezoelectric sensor, $\mathrm{S}=$ Sample.

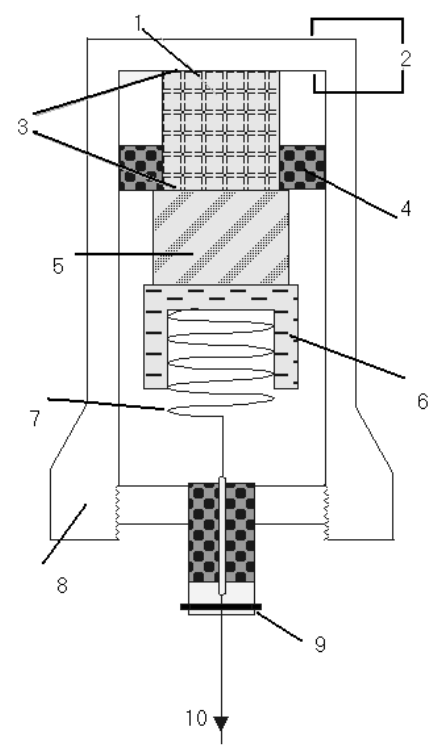

Figure 3: Scheme of microphone: 1) piezoelectric (PZT), 2) polished surfaces, 3) silicon grease, 4) hoop of Teflon ${ }^{\mathrm{TM}}$, 5) lead6) copper, 7) spring, 8) stainless steel, 9) BNC connector, 10) to oscilloscope.

Figure 4 shows a typical signal obtained without a Faraday cage. The oscilloscope was triggered by the photodetector as we explained before or by the noise generated by the discharge, with an extent of $5 \mu$ s. In this case, it is necessary to use a wave-guide in order to decouple the acoustic signal. We used a glass rod $8 \mathrm{~cm}$ long and since the sound speed in glass is $5450 \mathrm{~m} / \mathrm{s}$, the arrival time is about $14 \mu \mathrm{s}$, and this is a constant for all measurements. 
Another way to decouple the noise from the acoustic signal is by using thick samples, but depending on the kind of material, the attenuation can be strong. We recommend to use a glass rod where the attenuation is less than $15 \%$.

The acoustic coupling is very important for an efficient transmission of the acoustic energy. So it is recommendable to glue the sample, the wave-guide and the transducer together.

The amplitude of the acoustic signal is directly dependent of the energy and depends on the absorption coefficient as (1$10^{-\mathrm{A}}$ ), where A is the optical absorbance of the sample. So, high absorption assures high amplitude. If the front side of the sample reflects the laser wavelength, painting in black allows signal amplitude enhancement.

Here we first report the sound speed measurement obtained by a low energy laser, so, we are on the worst conditions.

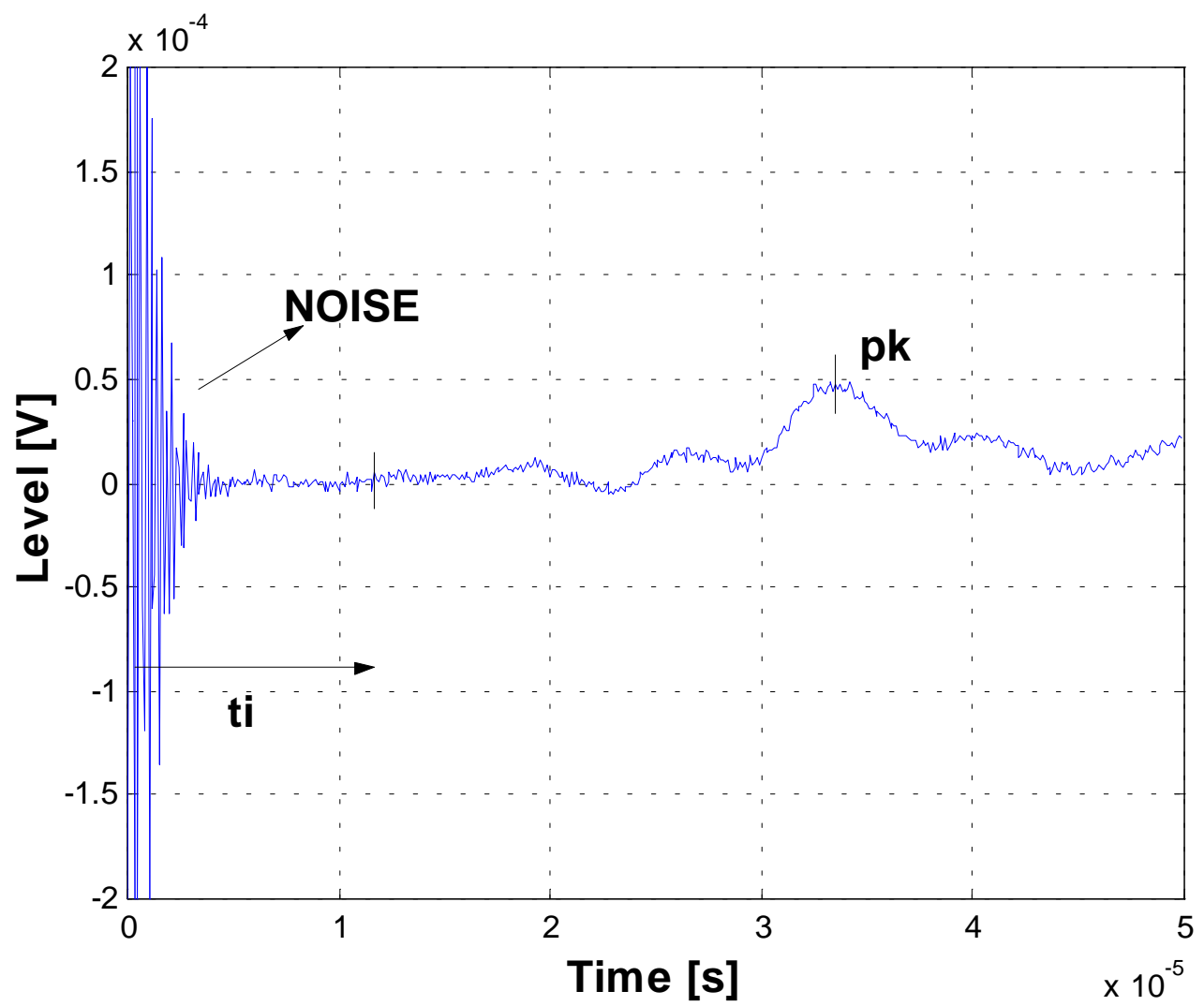

Figure 4. Photoacoustic signal. $\mathbf{t i}=$ arrival time, pk indicate the maximum amplitude for the second kind of measurements.

\section{RESULTS}

Table II shows the arrive times measured obtained by averaging 2000 pulses together with the thickness of the samples and the calculated speed of sound and the reported values found in the literature.

Observe that the agreement for homogeneous inorganic materials is quite good. The maximum deviation is presented for Aluminum where we have an error of $7.86 \%$. The Copper presents a smaller error (less than $1 \%$ ), due to the fact that $\mathrm{Cu}$ has good absorption properties in the UV, giving a high acoustic signal amplitude. For the organic materials, we did not find reported values, however, the obtained results are reasonable, taking into account that hard bone presents a porous region in the middle part, and that the soft bone is very flexible and the thickness was difficult to measure exactly. 
TABLE II. Results of measurements Photoacoustic.

\begin{tabular}{|c|c|c|c|c|}
\hline Material & Thickness [mm] & Arrival time [s] & $\begin{array}{c}\text { Calculated speed } \\
\text { of sound }[\mathrm{m} / \mathrm{s}]\end{array}$ & $\begin{array}{c}\text { Literature speed of } \\
\text { sound [m/s] }\end{array}$ \\
\hline Copper & $28.9 \mathrm{~mm}$ & $7.81 \mathrm{e}-6$ & 3701 & $3700^{*}$ \\
\hline Aluminum & $50.3 \mathrm{~mm}$ & $10.6 \mathrm{e}-6$ & 4745 & $5150^{*}$ \\
\hline Soft bone & $1.7 \mathrm{~mm}$ & $6.24 \mathrm{e}-7$ & 2724 & -- \\
\hline Hard bone $^{\text {TM }}$ & $4.0 \mathrm{~mm}$ & $1.408 \mathrm{e}-6$ & 2841 & --- \\
\hline Teflon $^{\mathrm{TM}}$ & $23.2 \mathrm{~mm}$ & $9.184 \mathrm{e}-6$ & 2526 & --- \\
\hline
\end{tabular}

* see reference [1]

On the other hand, if we want to improve the accuracy of the measurements, different thickness of the samples must be used. In this case we can measure the peak of the signal (see figure 4). As the length of the sample increases, the peak moves to longer times which can easily be measured. Figure 5 presents an example measured in Copper, where we have measured samples of 5 different thickness, detecting the arrive time of the first peak. In Figure 5 a graph of thickness vs arrival time is plotted, so from the slope of this curve the speed of sound can be obtained. In this method we found the same values reported with an uncertainty of $\pm 5 \mathrm{~m} / \mathrm{s}$.

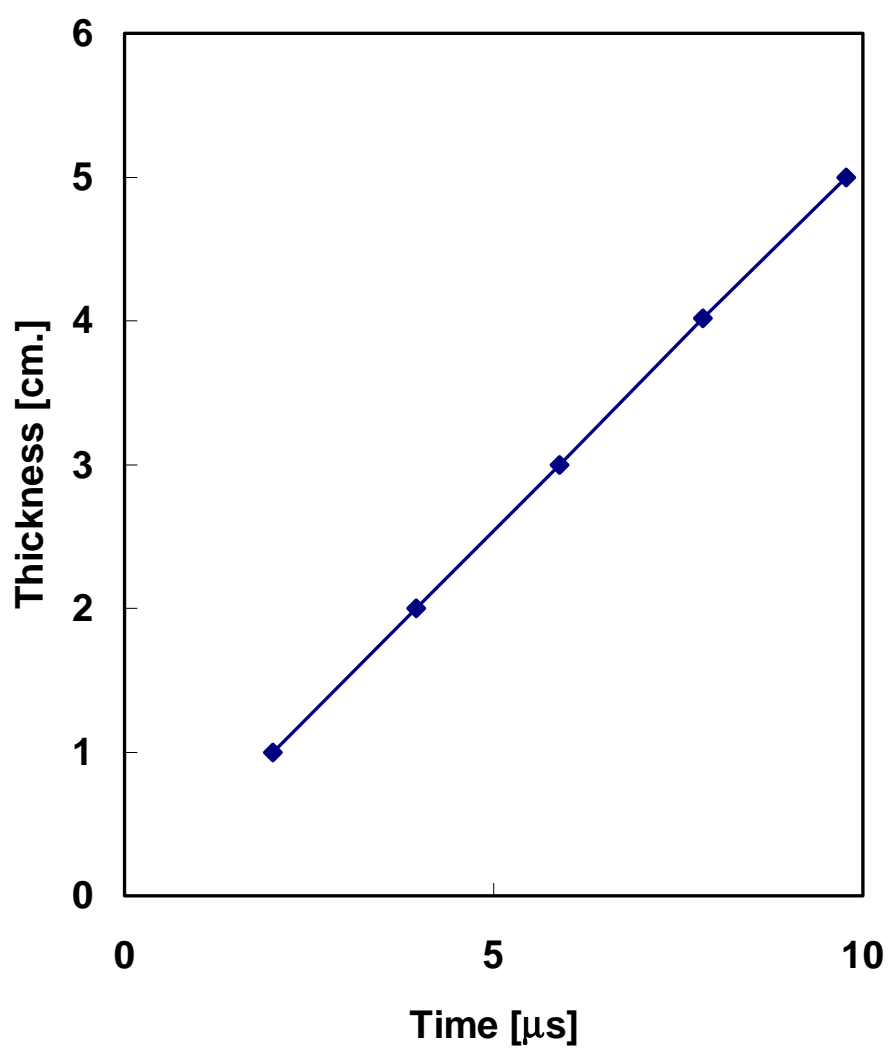

Figure 5. Peak amplitude arrival time as function of thickness sample, for $\mathrm{Cu}$ sample.

For these experiments it is only important to measure the thickness of the sample from the laser spot to the transducer, therefore thickness and size of the sample are not important. In these experiments we employed 3 homogeneous materials (Copper, Aluminum and Teflon ${ }^{\mathrm{TM}}$ in a cylindrical shape) and two no homogeneous organic materials (hard bone and soft 
bone in a irregular form). In the case of the no homogeneous materials, it is necessary to take into account the laser spot diameter. The spot must illuminate a broad area of the sample in order to obtain a global average of the sound speed in this material.

\section{CONCLUSIONS.}

We present a photoacoustic method as a versatile tool to measure the speed of sound in different materials. To generate the acoustic signal, a homemade Nitrogen laser was used. Good results were obtained in the determination of the arrival times of the photoacoustic signals for homogeneous inorganic materials. Also, in organic materials it is possible to obtain average values but with increased the uncertainty due to low level amplitudes of photoacoustic signals caused by absorption.

\section{ACKNOWLEDGMENTS.}

Financial support from the National Autonomous University of Mexico and the National Council of Science and Technology of Mexico is gratefully acknowledged. We would like to thank Dr. N. Bruce and H. Sobral for the critical reading of the manuscript.

\section{REFERENCES.}

1.-L. E. Kinsler, A. R. Frey, A. B. Coppens and J. V. Sanders, Fundamentals of Acoustic, p.461. John Wiley \& Sons, New York. 1982, Third Edition.

2.- A.G. Bell. "art. XXXIV on the production and reproduction of sound by light". Am. J. Sci. 20, 305-324, (1880)

3.- R. Castañeda-Guzmán, M. Villagran-Muniz, J-M. Saniger, O. Pérez-Martinez. " Photoacoustic phase transition of the ceramic $\mathrm{BaTiO}_{3}$ ". Appl. Phys. Letter. 73, 5, 623 (1998).

4.- R. Castañeda, M. Villagrán, J. M. Saniger, O. Pérez. "Detección Fotoacústica de transiciones de fase en cerámicas ferroelectricas”. Bol. Soc. Esp. Cerám. Vidrio. 38,5, 439 (1999).

5.- R. Castañeda Guzmán, M. Villagrán Muniz, J. Saniger Blesa, S.J. Pérez Ruíz and O. Pérez. "Photoacoustic analysis of the ferroelectric ceramics specific heat”. Appl. Phys. Lett. 77, 19,3087-3089 (2000).

6.- M. Villagrán M., M. Argote C. And C. Domínguez-Tagle P.,’Láseres de $\mathrm{N}_{2}$ para fines didácticos”, Revista Mexicana de Física, 40 (6), 935-945, (1994).

7.- M. Villagrán. "Láseres de colorantes para fines didácticos",. Rev. Mex. de Fis., vol. 41, No. 3, pag. 419-430, (1995).

8.- R. Castañeda Guzmán, S. J. Pérez Ruíz, M. Villagrán Muñiz, J. Saniger Blesa.. "Thermal Stability and Phase Transition by Photoacoustic Signal Analysis”. Analytical Sci.. 17, 122-125 (2001). 